REVISTA DE INVESTIGACIÓN

EN GESTIÓN CULTURAL

Córima, Revista de Investigación en Gestión Cultural

ISSN electrónico: 2448-7694

Universidad de Guadalajara

Sistema de Universidad Virtual

México

corima@udgvirtual.udg.mx

Año 6, número 11, julio-diciembre 2021

\title{
Mujeres y monumentos públicos en Chile: exclusión, corporalidad simbólica y deuda conmemorativa
}

\section{Women and public monuments in Chile: exclusion, symbolic corporality and commemorative debt}

\author{
Andrea Ortega ${ }^{1}$ \\ Pontificia Universidad Católica de Chile
}

https://doi.org/10.32870/cor.a6n11.7404

[Recibido: 18/3/2021; aceptado para su publicación: 15/5/2021]

\section{Resumen}

El presente artículo analiza la subrepresentación de la mujer en los monumentos públicos en Chile. En primer lugar, se examina de manera crítica la institucionalidad del Consejo de

\footnotetext{
${ }^{1}$ Doctora por la Universidad de Melbourne. Arquitecta y magíster en Desarrollo Urbano por la Pontificia Universidad Católica de Chile. Profesora del Diplomado en Patrimonio Cultural de la Pontificia Universidad Católica de Chile. Correo electrónico: aaortegaesquivel@uc.cl. ORCID: https://orcid.org/0000-0002-4988-085X

CÓMO CITAR ESTE ARTÍCULO

Ortega, A. (2021). Mujeres y monumentos públicos en Chile: exclusión, corporalidad simbólica y deuda conmemorativa. Córima, Revista de Investigación en Gestión Cultural, 6(11). DOI: 10.32870/cor.a6n11.7404
} 
Monumentos Nacionales y de la Comisión de Patrimonio Histórico, la cual autoriza la instalación y la intervención de los monumentos públicos en Chile. Desde esta institucionalidad se ha establecido un discurso patrimonial autorizado, con un enfoque monumentalista y eurocéntrico que, históricamente, ha anulado los discursos de las minorías y de los perdedores de las batallas, al imponer objetos conmemorativos con base en el juicio experto bajo un supuesto consenso social, desde y para la construcción de la identidad nacional. La metodología utilizada parte de la revisión del registro en línea del Consejo de Monumentos Nacionales durante el año 2020, y considera la figura femenina como un elemento escultórico y la identificación de la mujer como parte del valor simbólico o conmemorativo. Los resultados muestran que, de un total de 516 monumentos públicos, 67 (12.9\%) representan a la mujer de manera simbólica o conmemorativa.

\section{Palabras clave}

Discurso patrimonial autorizado; exclusión; monumento público; mujeres.

\section{Abstract}

This article analyzes the underrepresentation of women in public monuments in Chile. First, the institutionality of the National Monuments Council and the Historical Heritage Commission, which authorizes the installation and intervention of public monuments in Chile, are critically examined. From this institutional framework, an authorized heritage discourse has been established, with a monumentalist and Eurocentric approach, which has historically annulled the discourses of minorities and the losers of battles, by imposing commemorative objects based on expert judgment, under a supposed social consensus, from and for the construction of national identity. The methodology used considers the review of the online registry of the Council of National Monuments during the year 2020, and considers the female figure as a sculptural element and the identification of women as part of the symbolic or commemorative value. The results show of a total of 516 public monuments, 67 (12.9\%) represent women in a symbolic or commemorative way.

\section{Keywords}

Authorized heritage discourse; exclusion; public monument; women. 


\section{Introducción: la figura femenina en los monumentos públicos y la exclusión de la mujer en la historia oficial de las naciones}

Los monumentos públicos (MP) son objetos instalados en los espacios públicos que buscan transmitir contenidos ideológicos (Vega, 2016). Su presencia es habitual en los espacios públicos centrales de las ciudades, desde los cuales reproducen un discurso "sutilmente repetitivo, mediante [...] poses, 'estetizadas' jerarquías y discriminaciones de género" (Vega, 2016, p. 1). Los MP son depositarios de la memoria de ciertos personajes o acontecimientos, a la vez que influyen determinantemente en la forma en que estos son percibidos, generando sentido (Vega, 2016).

Durante el proceso de construcción de las naciones modernas del siglo XIX, las élites volvieron su gusto hacia las formas clásicas que representan el origen de la democracia, al idealizar la figura de mujeres desnudas e instalar esculturas en los espacios arquitectónicos de las naciones emergentes (Vega, 2016); sin embargo, esta presencia de figuras femeninas no implicó la inclusión de las mujeres en los cargos de poder, sino al contrario: las mujeres han sido sistemáticamente excluidas y subrepresentadas en los espacios donde se toman las decisiones políticas de las naciones (Yuval-David, 2004; Vega, 2016).

La figura femenina se manifiesta muchas veces en los MP y en las estatuas pero, en general, no son las mujeres las que están representadas, sino su corporalidad, que simboliza alegóricamente ideas abstractas (la libertad, la justicia, la naturaleza, la patria, etcétera) y sentimientos (bondad, caridad, ternura, etcétera), lo que destaca la relación entre ser mujer y ser madre (Bulbeck, 1992; Vega, 2016). La proliferación de este tipo de esculturas figurativas en los espacios públicos no ha estado determinada por meros criterios estéticos, ni para conmemorar eventos históricos, sino para para ejercer una función educativa que busca transmitir ciertos valores de las élites políticas (Gas Barrachina, 2020).

La corporalidad simbólica del cuerpo femenino se alza en representación de los valores que buscan contribuir a la conformación de la identidad nacional (Zárate, 2003; Gas Barrachina, 2020). En este contexto, la abundancia de los MP que reflejan cuerpos femeninos para embellecer y simbolizar ideas de forma alegórica contrasta 
con la exclusión sistemática de su presencia en la política y su invisibilización en el relato patriarcal de la historia oficial de las naciones.

Desde la segunda mitad del siglo XIX, el urbanismo en América se ha planteado, en gran medida, a través de la configuración de los espacios públicos como lugares de memoria vinculados al relato de la historia oficial de las naciones, en donde destacan los próceres masculinos de la independencia y acontecimientos relevantes reflejados a través de "monumentos al progreso" (Gutiérrez, 2004, p. 77). Estos MP comprenden obras ornamentales y mobiliario urbano, los cuales muchas veces, con fines casi meramente ornamentales, promueven la exaltación de una imagen asociada a las ideas de progreso y de victoria desde las élites políticas masculinas (Gutiérrez, 2004).

Por otro lado, los centenarios de las independencias de Argentina, Chile (ambas en 1910) y Perú (1921) desarrollaron una serie de estrategias políticas que consideraron la instalación de MP con el fin de simbolizar las alianzas establecidas y fortalecerlas (Monteverde, 2017). Por ejemplo, el gobierno argentino erigió un MP dedicado al prócer chileno Bernardo O'Higgins en Buenos Aires, a modo de retribución por el MP que el gobierno chileno construyó en Santiago en honor a José de San Martín en 1862 (Monteverde, 2017). En este contexto, son menos comunes los MP que conmemoran a las mujeres, ya que el objetivo era conmemorar las hazañas de los hombres próceres de la patria.

En el marco europeo y anglosajón, desde la Primera Guerra Mundial los MP han sido utilizados sistemáticamente por las autoridades para recontextualizar las realidades de la guerra, lo que fomenta discursos de celebración de la nación y el noble sacrificio de los soldados (Abousnnouga y Machin, 2011). Si bien gran parte de la forma en que las mujeres participaron en la guerra se ha omitido en los MP, la representación de la figura femenina ha sido clave en la legitimación de un discurso particular de la guerra, la cual se ha recontextualizado y resignificado a través del uso de la figura femenina. Por otro lado, en la sociedad británica se conmemora la muerte de "nuestros muchachos" (our boys), por los hombres jóvenes asesinados en Afganistán (Abousnnouga y Machin, 2011). 
En el caso de Australia, los ideales abstractos representados en las figuras femeninas a través de los MP existen según un registro nacional realizado entre 1987 y 1989. Bulbeck (1992) realizó un "Registro Nacional de Monumentos Inusuales" erigidos a los "otros" australianos (aborígenes, inmigrantes no anglosajones, obreros y mujeres) y a la gente "común" (en contraposición a los conquistadores y líderes políticos). Definió estas categorías desde las referencias de la historia obrera, la historia de la mujer y la historia aborigen de Australia (Bulbeck, 1992).

En este contexto, Bulbeck registró los MP referidos a las "mujeres alegóricas". Aunque las mujeres durante el siglo XIX no participaban directamente las guerras, ni ejercían cargos políticos importantes, la figura femenina se utilizó sistemáticamente para representar las virtudes a las que aspiraban los hombres, vinculándolas principalmente a su proximidad con la naturaleza y como símbolo de victoria.

Recientemente, han surgido MP dedicados de modo amplio a la mujer, lo que incluye memoriales inaugurados con actos en homenaje a las víctimas de violencia de género, como el caso de España (Vega, 2016). En este contexto, las organizaciones y las prácticas feministas recientes han buscado deconstruir las estructuras patriarcales al cuestionar las normas culturales existentes y al exigir una mayor inclusión de la mujer en los distintos ámbitos sociales (Nead, 2013).

Sin embargo, es importante que la reciente inclusión conmemorativa de las mujeres, en materia de los MP, sea analizada de manera crítica. Por ejemplo, según Vega (2016), los MP dedicados a las mujeres trabajadoras en España siguen representando, de manera general, las labores domésticas históricamente feminizadas bajo un agradecimiento nostálgico por los servicios prestados (como las cocineras, las vendedoras de castañas, las queseras, etcétera).

Así, es necesario promover un enfoque crítico para construir una historia oficial más inclusiva y que represente mejor a las mujeres, lo que incluye su visualización en los valores conmemorativos y la proyección de ideales sociales bajo un enfoque de igualdad de género a través de los MP (Kenna y Waters, 2016; Casey, 2010). Aunque la forma y la frecuencia en la que la historia oficial representa a las mujeres ha mejorado a lo largo de las décadas, todavía necesita refinarse la mirada para que esta consideración sea realmente equitativa y que se entienda el desequilibrio y la 
subrepresentación de las mujeres más allá de los textos escritos, como por medio de los discursos representados a través de los MP y los memoriales (Kenna y Waters, 2016).

Al considerar el contexto nacional e internacional sobre las mujeres en los MP, el presente artículo analiza de manera crítica cómo la mujer ha sido excluida y subrepresentada en los MP en Chile. Esta discusión ha salido a la luz pública de manera evidente a partir del reciente estallido social, o revuelta popular, originada en Santiago y luego en otras ciudades chilenas (Gran Valparaíso, Gran Concepción, Arica, Iquique, Antofagasta, La Serena, Rancagua, Chillán, Valdivia, Osorno, Puerto Montt y Punta Arenas), desde octubre de 2019 hasta febrero de 2020. Desde entonces, se manifestó una serie de acciones iconoclastas sobre los MP en los espacios públicos, como protesta en contra de la historia oficial (Urrejola, 2019).

Márquez (2020) enmarca estas acciones como "reivindicaciones en contra del abuso del modelo social y económico imperante" (p. 3), es decir, en contra de la imposición del modelo neoliberal. La "monumentalidad patria" se transformó, de un escenario para los próceres de la nación, a nuevos MP que se travistieron con graffitis, consignas y banderas que aludieron a los grupos sociales históricamente marginados en Chile, lo que incluye los pueblos originarios (particularmente el pueblo Mapuche), las diversidades sexuales y de género, entre otros grupos (Márquez, 2020).

Las consignas descolonizadoras no solo se manifestaron en la central Plaza Italia, actualmente denominada popularmente como Plaza de la Dignidad en Santiago, sino también en otros espacios públicos de la ciudad de Santiago y en otras ciudades chilenas.

De esta manera, entre otros grupos sociales y causas involucradas, el movimiento feminista logró manifestarse de forma visible en los espacios públicos a través de las demandas y los cuestionamientos sobre los MP de los colonizadores y los héroes patrios, con lo que se cuestionó, incluso, la manera en la que las mujeres usan e intervienen en el espacio público de las ciudades latinoamericanas (Fleischer y Marín, 2019). 
A continuación, se presenta el concepto de MP en la actual Ley $N^{\circ} 17.288$ de Monumentos Nacionales en Chile y cómo el discurso patrimonial autorizado ha operado desde el Consejo de Monumentos Nacionales (CMN).

\section{El discurso patrimonial autorizado de los monumentos públicos en Chile}

Según la Ley $N^{\circ} 17.288$ de los Monumentos Nacionales (MN), en el título IV, "De los Monumentos Públicos", artículo 17, los monumentos públicos (MP) son los objetos (estatuas, columnas, fuentes, pirámides, placas, coronas e inscripciones) "que estuvieren colocados o se colocaren para perpetuar memoria en campos, calles, plazas y paseos o lugares públicos", los cuales quedan bajo la tuición del Consejo del CMN (Ley No $17.288,1970$, p. 6).

Los MN en la categoría de MP no requieren declaración expresa mediante decreto o ley para ser considerados como tales, ya que, en virtud de lo consignado en el artículo 21 de la Ley $N^{\circ} 17.288$, son MN en la categoría de MP todos los objetos antes mencionados instalados para perpetuar la memoria en los espacios públicos, sin perjuicio de que para su instalación o traslado se deba solicitar la autorización previa del CMN.

Los MP están ubicados en los espacios públicos con el objetivo de conmemorar acontecimientos, individuos o grupos de personas que han influido en la cultura e historia nacional, razón por la cual, en muchos casos, son hitos del paisaje urbano y referencias espaciales en el contexto de las ciudades chilenas (Consejo de Monumentos Nacionales, s. f.). Los MP no requieren de una declaración expresa por decreto para ser reconocidos, sino que son definidos por el ministerio de la Ley $\mathrm{N}^{\circ}$ 17.288.

Dentro del CMN, el Área de Patrimonio Histórico se encarga de velar por la protección y puesta en valor de los monumentos históricos, así como de llevar el registro de los museos. Esta área coordina y participa en la Comisión de Patrimonio Histórico, la cual se encarga de estudiar y autorizar solicitudes de las instalaciones, los traslados, las intervenciones o las restauraciones de los MP. 
La Comisión de Patrimonio Histórico está compuesta por consejeros del Instituto de Conmemoración Histórica, de la Sociedad Nacional de Bellas Artes, de la Asociación de Pintores y Escultores de Chile, del Museo Histórico Nacional, del Museo Nacional de Bellas Artes, del Archivo Nacional, de la Sociedad de Escritores de Chile y de la Sociedad Chilena de Historia y Geografía (Consejo de Monumentos Nacionales, s. f.). De esta manera, en Chile los MP están definidos por expertos de gremios autorizados y representativos de la institucionalidad cultural del estado.

De acuerdo con la institucionalidad actual del CMN y la Ley de Monumentos Nacionales en Chile, los MP están definidos desde un enfoque monumentalista y eurocéntrico. Según el enfoque teórico propuesto por la arqueóloga australiana Laurajane Smith, esta institucionalidad y legislación promueve un discurso patrimonial autorizado (DPA, Authorized Heritage Discouse [AHD]) a través de un relato oficial y hegemónico que depende de las afirmaciones de los grupos de poder y del conocimiento de los expertos, los técnicos y las autoridades políticas. Estos actores son quienes definen, decretan o institucionalizan valores estéticos específicos bajo el sustento de agencias culturales estatales y privadas (Smith, 2006).

EI DPA se transmite a través de narrativas grandilocuentes de la nación, desde el juicio estético y la experiencia técnica (Smith, 2006), y privilegia la monumentalidad e impone la significancia intrínseca del objeto o sitio con base en el juicio experto, un supuesto consenso social y la construcción de la identidad nacional (Smith, 2006). En este contexto, el DPA promueve la cosificación y la monumentalización del patrimonio cultural junto a un discurso respaldado por políticas internacionales definidas por la UNESCO y apoyadas por la Convención sobre el Patrimonio Mundial de 1972 (UNESCO, 1972; Smith, 2011).

Además, el DPA se impone sobre patrimonios ocultos o indeseados (Tunbridge y Ashworth 1996; Smith, 2011). En este sentido, el patrimonio es algo que va más allá de aquellos monumentos dedicados a las memorias victoriosas de la historia, debido a que también existe patrimonio en los protagonistas anónimos, en las resistencias y en los perdedores (Criado-Boado y Barreiro, 2013). De esta manera, el patrimonio es intrínsecamente un proceso conflictivo, por lo cual en todo desarrollo 
de patrimonialización existirían sujetos que buscan imponer discursos hegemónicos para anular otros discursos (Criado-Boado y Barreiro, 2013).

La institucionalidad actual que define los MP en Chile no está establecida desde el concepto de patrimonio, sino a partir de un DPA monumentalista que anula los discursos de las minorías y se establece como aquello que es intrínsicamente valioso para ser protegido. En contradicción a las definiciones y prácticas tradicionales del patrimonio monumentalista de occidente, Smith (2006) plantea que el patrimonio no es una "cosa", sino que el patrimonio se comprende de mejor manera como una práctica cultural y una construcción social que involucra una amplia diversidad de valores. La construcción discursiva del patrimonio es parte de los procesos culturales de una sociedad, mientras que la práctica de la conservación del patrimonio se establece a través de técnicas y procedimientos de gestión definidos por administradores y expertos del patrimonio (Smith, 2006).

En la siguiente sección se presenta la metodología utilizada para desarrollar el análisis sobre la representación de la mujer en los MP en Chile.

\section{Metodología}

Se realizó un análisis sobre la representación de la mujer en los MP en Chile con base en la revisión del registro de los MN publicados en la página web del CMN, y se consideró específicamente la lista pública de los MP durante el año 2020 (Consejo de Monumentos Nacionales, s. f.). Esta lista corresponde a un registro parcial, ya que, al momento de realizar el estudio, el CMN no contaba con un registro total de los MP del país. Esta base de datos parcial y en construcción se basó en el libro Chile a través de sus monumentos públicos (dividido en norte, centro y sur), que surgió a raíz de un catastro realizado por el CMN en las capitales provinciales del país, excepto las capitales provinciales de la Región Metropolitana de Santiago².

2 Solicitud: uso de imágenes de monumentos públicos en artículo académico. [info@monumentos.gob.cl]. 11 de septiembre de 2020. Info - Consejo de Monumentos Nacionales, 
Sin embargo, luego de realizar este análisis, el CMN publicó en marzo de 2021 el primer estudio de monumentos públicos a mujeres en capitales regionales de Chile, el cual excluye a "todos aquellos MP en los que aparece una o varias esculturas femeninas con funciones estéticas o simbólicas" (Consejo de Monumentos Nacionales, 2021, p. 6). Es importante considerar lo anterior, ya que el análisis presentado en este artículo sí considera aquellos MP que representan esculturas femeninas con funciones estéticas o simbólicas, las cuales se presentan cuantitativamente y se analizan cualitativamente.

Para comenzar el análisis, se realizó una búsqueda preliminar de la palabra "mujer" y "madre". Con este primer registro, se eliminaron aquellos MP en los que se menciona a la madre del personaje masculino conmemorado en la biografía incluida en la ficha técnica (por ejemplo, en las fichas técnicas se menciona sin mayor detalle a la madre de Bernardo O'Higgins, Padre Hurtado, Pablo Neruda, Andrés Sabella, etcétera). Luego, se realizó una revisión al total de fichas técnicas que incluyen fotografías de los MP, donde se observó que la figura femenina fuera parte del MP como elemento escultórico. A partir de esta revisión, se elaboró una matriz que incluyó información básica de cada MP (nombre de la obra, año de inauguración y lugar), la descripción de la figura femenina, el valor conmemorativo de la obra y una fotografía.

\section{Resultados}

De un total de 516 MP registrados por el CMN, 67 de ellos (12.9\%) considera la figura femenina como un elemento simbólico-escultórico o como parte del valor conmemorativo. En 17 de ellos, la mujer solo se representa como un elemento simbólico-escultórico. En otras palabras, del total de 516 MP registrados por el CMN, solo 50 representan a la mujer como parte de su valor conmemorativo (9.6\%). Esto demuestra la baja presencia de la mujer en la conmemoración oficial de los acontecimientos que han influido en la historia nacional de Chile.

Servicio Nacional del Patrimonio Cultural Ministerio de las Culturas, las Artes y el Patrimonio, Gobierno de Chile. 


\section{La figura femenina como elemento simbólico-escultórico: la virginidad, la fertilidad, la tierra, la patria y la victoria}

De un total de 516 MP, 17 representan a la figura femenina únicamente como un elemento simbólico-escultórico. A este número es necesario sumarle los cinco MP que conmemoran a la Virgen María a través de su representación en la Virgen de la Candelaria y la Virgen del Carmen ${ }^{3}$, como advocación mariana de la religión católica. La figura femenina simboliza la fertilidad en dos MP, "La Vendimia" de Curicó y "Madre Tierra" en Talca. En el primero, la mujer representa la fertilidad y la voluptuosidad, propia de la faena de la refriega, y en el segundo, la escultura representa a una mujer que emerge de la tierra y lleva en sus brazos espigas de trigo y racimos de uvas, lo que conmemora el Bicentenario de la Independencia de Chile. La victoria también es un valor simbolizado a través de la figura femenina, por ejemplo, en "Escultura de la paz mundial", erigida en Copiapó en 2011, la cual conmemora el rescate de los 33 mineros de la mina San José en 2011.

\section{La mujer en el valor conmemorativo: la prócer, la madre, la mujer común y la víctima}

En 50 MP se considera a la mujer como parte de su valor conmemorativo. En primer lugar, existen 20 MP dedicados a la prócer y poeta Gabriela Mistral, y seis dedicados a otras mujeres. Por otro lado, existen dos MP que vinculan directamente a la mujer con la maternidad. La escultura "A la mujer", ubicada en Antofagasta, es un homenaje a las mujeres y la crianza, es decir, se vincula el ser mujer con ser madre. Al mismo tiempo, conmemora la historia de la lucha por los derechos de las mujeres en Chile, lo que indica que esta es una "referencia obligatoria" (Consejo de Monumentos Nacionales, s. f.). "Mujer pampina", en Pozo Almonte, es una escultura

\footnotetext{
3 "Ofrenda a la Virgen de la Candelaria" de Copiapó, "María virgen madre de Dios y Reina del Carmelo" de La Serena, "Virgen del Carmen" de Antofagasta, "Virgen del Carmen" de Ovalle, y "María Virgen Madre de Dios y Reina del Carmelo" de La Serena.
} 
de yeso que representa a una mujer con dos hijos, uno en sus brazos y el otro de la mano.

Asimismo, existen algunos MP que hacen homenaje a la mujer común. Por ejemplo, "Alma del pueblo", en Antofagasta, en la cual se representan dos mujeres, tres hombres y un niño de la mano de una de las mujeres. De esta manera, se manifiesta el vínculo entre la mujer, la madre y la crianza.

Otro simbolismo y motivo conmemorativo es la mujer como víctima. Por un lado, en términos simbólicos, destaca la "Lloradora del Cementerio Municipal" en Copiapó, una escultura de una mujer con velo y toga, que tiene la mirada perdida hacia el cielo, que conmemora la histórica tradición de las lloradoras o lamentatrices, que eran mujeres contratadas para llorar en los funerales de personas connotadas.

Por otro lado, en términos conmemorativos, el "Lugar de memoria nunca más" es un memorial en forma de monolito, inaugurado en Iquique en 2015 , dedicado a los presos políticos de la dictadura militar en el Regimiento de Telecomunicaciones de la VI, que funcionó como un centro de detención y torturas, donde se encontraron 70 mujeres.

Cabe comentar que también existen MP que mencionan a las mujeres, pero no representan escultóricamente a la figura femenina. Por ejemplo, el "Homenaje a los trabajadores de la pampa salitrera", inaugurado en 1989 en Pozo Almonte, conmemora a las familias obreras reprimidas con violencia por el ejército chileno en la masacre de la Escuela Santa María de Iquique en 1907, donde murieron miles de hombres, mujeres y niños de la pampa salitrera.

\section{Gabriela Mistral: bustos y otras representaciones}

Del total de $67 \mathrm{MP}$ registrados por el CMN que simbolizan o conmemoran a la mujer, 20 están dedicados a Gabriela Mistral, es decir, 29.8\%. De los 20 MP, doce corresponden a bustos de Gabriela Mistral ubicados en diversas ciudades del territorio chileno (Arica, Iquique, Copiapó, Illapel, La Serena, Linares, Chillán, Osorno, Puerto Mont, Puerto Aisén, Punta Arenas y Cabo de Hornos). El MP dedicado a la Premio 
Nobel de Literatura emplazado en Punta Arenas fue inaugurado en 1959 en homenaje a las labores de la poeta y profesora normalista en la dirección del Liceo de Niñas (Consejo de Monumentos Nacionales, s. f.).

En las fichas técnicas de cuatro de los doce bustos se indica que "diversas corrientes del feminismo y la literatura hoy la reivindican desde su lesbianismo, la promoción de la educación de las mujeres y el hito que implicó su ejemplo, al ser la primera mujer en obtener importantísimos reconocimientos mundiales" (Consejo de Monumentos Nacionales, s. f.). Entre otros MP en honor a Gabriela Mistral, se encuentra el "Libro de Bronce", en homenaje a los poetas y escritores de Chile, lo que incluye a la poeta en La Serena, y una escultura rodeada por tres niños en posición de una ronda, en Antofagasta.

\section{Otras mujeres con nombre y apellido}

Además de los MP dedicados a Gabriela Mistral, existen seis mujeres representadas y conmemoradas en el registro de MP del CMN: Lenka Franulic Zlatar (Antofagasta), Sor Ana Giglia Zappa (Antofagasta), María Isabel Peralta (La Serena), Mónica Donoso (Curicó), Amelia Troncoso (Linares) y Alfonsina Storni (Punta Arenas).

Mónica Donoso fue una de las fundadoras de Curicó, quien donó partes de sus terrenos para emplazar y trazar la Plaza de Armas; Amelia Troncoso fue una maestra de Linares que educó a varias generaciones de linarenses y fundó un colegio que lleva su nombre en 1919. Por su parte, Alfonsina Storni fue una poeta de avanzada en la escena lírica, inmigrante suiza radicada en Argentina e influenciada por el modernismo de mediados de la década de 1910.

Lenka Franulic fue la primera mujer reconocida con el Premio Nacional de Periodismo en 1957, y su MP es un homenaje por parte de la Universidad Técnica del Estado, sede Antofagasta. Por su parte, Sor Ana Giglia fue una religiosa de la Congregación de las Hijas de Santa Ana, fundada en 1893, que impulsó la educación en la ciudad de Antofagasta. Por último, María Isabel Peralta fue una poeta nacida en Paihuano, quien en 1933 publicó de manera póstuma el libro Caravana parda, que recopila toda su poesía publicada, prologado por Gabriela Mistral. 


\section{Conclusiones}

El análisis a los MP registrados por el CMN revela una evidente representatividad baja de la mujer como parte del valor conmemorativo ( $9.6 \%$ del total). Esto demuestra la exclusión de las mujeres en el DPA en Chile y la deuda existente en su consideración en los valores conmemorativos de los MP reflejados en la historia oficial de la nación.

EI DPA en Chile privilegia la monumentalidad y la construcción de la identidad nacional a través de un relato hegemónico de próceres masculinos de la independencia. Con la revuelta popular en Chile, las acciones iconoclastas han reflejado un descontento en cómo se ha materializado la representación de la historia oficial de la nación en los MP. En este contexto, el DPA, reflejado a través de los MP, ha sido cuestionado a través de diversas intervenciones feministas, entre otros grupos sociales sistemáticamente excluidos. Más allá de condenar estas manifestaciones y acciones iconoclastas en contra de los MP en los espacios públicos de las ciudades chilenas, pueden entenderse como una oportunidad desde su potencial de transformación.

Los procesos de resignificación del patrimonio cultural son una oportunidad para ampliar y diversificar la mirada sobre las representaciones simbólicas de aquellos elementos que establecen relatos sobre la identidad de las naciones. En este contexto, la exclusión y la deuda conmemorativa de la mujer en el relato oficial y la representación de los cuerpos femeninos como mero objeto contemplado refleja no solo el carácter político del patrimonio, sino la violencia simbólica del relato hegemónico de los MP en Chile.

A pesar de que el DPA de los MP en Chile aún refleja un enfoque primordialmente patriarcal, es importante destacar el incipiente reconocimiento de las mujeres. Dentro de este registro, Gabriela Mistral es representada en casi $30 \%$ del total, y gran parte de las fichas técnicas del CMN no están enfocadas solo en su obra literaria, sino en su rol en la promoción de la educación de las mujeres. Además, se reivindica su lesbianismo como parte de su identidad, lo que refleja una incipiente inclusión del discurso feminista y de las diversidades sexuales. 
Asimismo, cabe destacar la identificación de otras seis mujeres en el registro de los MP, las cuales son originarias de diversas localidades del territorio nacional y son destacadas por su trabajo en diversos ámbitos del desarrollo cultural local. Estas últimas representaciones abren la posibilidad de continuar con el avance de la descentralización y la diversificación de los valores simbólicos representados en los MP en Chile.

Finalmente, este artículo es un llamado a reflexionar sobre las implicaciones concretas de la exclusión de las mujeres en el DPA y la historia oficial de la nación, lo cual ha influido en la representación del simbolismo del cuerpo femenino desde su mera función escultórica y en la deuda conmemorativa del total de los MP presentes en el territorio nacional. Este asunto no solo concierne a Chile, sino que debería ser estudiado en el resto de los países latinoamericanos.

Las manifestaciones recientes en Latinoamérica han evidenciado un incipiente proceso de cuestionamiento a la estructura patriarcal, al mismo tiempo que han surgido intervenciones en los espacios públicos y en los MP desde un espíritu descolonizador. Más allá de comprender estas acciones únicamente como hechos vandálicos, surge la oportunidad de repensar no solo lo que reflejan los MP, sino las estructuras sociales y las políticas imperantes de las naciones colonizadas, donde persisten hegemonías que no son representativas de la diversidad cultural presente

\section{Referencias}

Abousnnouga, G. y Machin D. (2011). Visual discourses of the role of women in war commemoration: A multimodal analysis of British war monuments. Journal of Language and Politics, 10(3), 322-346. https://doi.org/10.1075/jlp.10.3.02abo

Bulbeck, C. (1992). Women of Substance: The Depiction of Women in Australian Monuments. Hecate, 18(2). Recuperado de: https://search.informit.org/doi/abs/10.3316/ielapa.930505131

Casey, J. G. (2010). The American pioneer woman circa 1930: Cultural debates and the role of public art. American Studies, 51(3/4), 85-107. 
Consejo de Monumentos Nacionales. (s. f.). Monumentos públicos. Consejo de Monumentos Nacionales. Recuperado de: https://www.monumentos.gob.cl/monumentos/monumentos-publicos

Consejo de Monumentos Nacionales. (2021). Estudio de Monumentos Públicos a Mujeres en capitales regionales de Chile. Consejo de Monumentos Nacionales. Recuperado de: https://www.monumentos.gob.cl/publicaciones/articulos/1er-estudiomonumentos-publicos-mujeres-capitales-regionales

Criado-Boado, F. y Barreiro, D. (2013). El patrimonio era otra cosa. Estudios atacameños, (45), 5-18.

Fleischer, F. y Marín, M. (2019). Atravesando la ciudad. La movilidad y experiencia subjetiva del espacio por las empleadas domésticas en Bogotá. Revista Eure, 45(13).

Gas Barrachina, S. (2020). La historia fragmentada de los monumentos públicos: el proceso de identificación y su significado en la actualidad. Arte y políticas de identidad, 23(23), 182-198. Recuperado de: https://doi.org/10.6018/reapi.461221

Gutiérrez, R. (2004). Monumento conmemorativo y espacio público en Iberoamérica. Madrid: Cátedra. Recuperado de: http://www.ugr.es/ rgutierr/PDF2/LIB\%20011.pdf

Kenna, J. y Waters, S. (2016). Women on America's Historical Landscape: Teaching with Monuments and Memorials. Social Studies Research and Practice, 11(3). Recuperado de: http://www.socstrpr.org/wpcontent/uploads/2017/01/MS06698-Kenna-et-al.pdf

Ley $\mathrm{N}^{\circ} 17.288$ de 1970. Legisla sobre monumentos nacionales; modifica las leyes 16.617 y 16.719 ; deroga el decreto ley 651 , de 17 de octubre de 1925. Recuperado

de: https://www.bcn.cl/leychile/navegar?idNorma $=28892$

Márquez, F. (2020). Por una antropología de los escombros. El estallido social en Plaza Dignidad, Santiago de Chile. Revista 180, 45, 1-13. Recuperado de:

http://www.revista180.udp.cl/index.php/revista180/article/view/717/4 $\underline{38}$

Monteverde, R. (2017). Política internacional de la posguerra del Pacífico, remodelación urbana y proyectos escultóricos de Lima: el monumento público a Francisco Bolognesi y los Caídos en la Batalla de Arica (1905). 
Historia (Santiago), 50(2),

663-697. https://dx.doi.org/10.4067/s0717-71942017000200663

Nead, L. (2013). El desnudo femenino: arte, obscenidad y sexualidad. Madrid: Tecnos.

Smith, L. (2006). Uses of Heritage. Londres: Routledge.

Smith, L. (2011). El "espejo patrimonial", ¿ilusión narcisista o reflexiones múltiples? Antípoda, 12, 262.

UNESCO. (1972). Convention Concerning the Protection of the World Cultural and Natural Heritage. Recuperado de: http://whc.unesco.org/archive/convention-en.pdf

Tunbridge, J. y Ashworth, G. (1996). Dissonant Heritage: The Management of the Past as a Resource in Conflict. Chichester: John Wiley and Sons Ltd. Urrejola, J. (2019). Chile: destrucción de monumentos como protesta contra la historia oficial. DW. Recuperado de: https://www.dw.com/es/chiledestrucci\%C3\%B3n-de-monumentos-como-protesta-contra-lahistoria-oficial/a-51202577

Vega, E. (2016). ¿Una violencia invisible? Las mujeres en los monumentos públicos. Boletín de Arte, 37, 213-225. Universidad de Málaga: Departamento de Historia del Arte.

Yuval-David, N. (2004). Género y Nación. Lima: Flora Tristán.

Zárate, V. (2003). El papel de la escultura conmemorativa en el proceso de construcción nacional y su reflejo en la ciudad de México. Historia Mexicana, 53(2), 417-446. 